\title{
Very short-term load forecasting based on a pattern ratio in an office building
}

\author{
Ah-Yun Yoon, Hyeon-Jin Moon, Seung-Il Moon* \\ Department of Electrical and Computer Engineering, Seoul National Univ. \#013, 1 Gwanangno, Gwanak-gu, Seoul, 08826, \\ South Korea
}

\begin{abstract}
The pattern of electric demand needs to be analyzed to obtain a simple and precise Very Short-Term Load Forecast (VSTLF) for an office building because the electric demand of a small power system such as a building is difficult to express as a function. In order to develop an improved VSTLF, data from LG Electronics was analyzed. The proposed method is compared to the conventional method using a correlation between electric demand and temperature. The test results show that the proposed method based on a pattern ratio is better than the conventional method based on linear regression. MAPE of the proposed method is $9.0973 \%$, while MAPE of the conventional method is $9.4533 \%$.
\end{abstract}

Keywords: Very short-term load forecasting, office building, pattern, temperature

\section{Introduction}

The number of large-sized office buildings in South Korea (hereafter, Korea) is increasing. If electricity consumption increases in buildings on the peak time, the electricity bill becomes more expensive under the Time-of-Use (TOU) price system in Korea. Consequently, building owners have installed an Energy Storage System (ESS) in the building to reduce the electricity bill [1]. A Building Energy Management System (BEMS) is necessary to operate ESS economically. The BEMS can control an ESS to reduce peak load. Thus, Short-Term Load Forecasting (STLF) is very important.

There are many studies on STLF. Tracing the history, we can classify STLF studies under two methods: statistical analysis and artificial intelligence. The statistical analysis method includes exponential smoothing, regression analysis, and time series analysis. The artificial intelligence method includes fuzzy model, expert systems, and artificial neural networks. In the statistical analysis method, a functional relationship between electricity consumption and weather variables is intuitive. However, a nonlinear relationship between variables is difficult to describe. In the artificial intelligence method, a nonlinear relationship between variables is expressed easily. However, variables that have a special relationship with electricity consumption are difficult to cover by this method [2]. Nevertheless, it is difficult to address that the artificial intelligence model is superior to the statistical analysis method [3][5]. In [5], six forecasting models are compared. Exponential smoothing for double seasonality shows the best performance for forecasting Brazil's electricity consumption. Most studies have researched STLF for a large power system [3]-[10]. Recently, researchers have developed interests in STLF for buildings [1], [11]-[18]. There are a few studies in the literature on the STLF of an office building for the purpose of increasing efficiency [18], [19]. There have been no studies on the STLF of an office building with only electricity consumption [19]. Therefore, this paper focuses on the STFL of an office building, which seems to be a worthwhile subject to investigate in terms of the installation of an ESS in an office building.

\footnotetext{
* Manuscript received December 1, 2015; revised April 5, 2016.

Corresponding author. Tel.: +82-10-5765-2280; E-mail address: ah.yun924@gmail.com

doi: $10.12720 /$ sgce.5.2.94-99
} 
This paper analyzes a building under a TOU price system in Korea. The time unit of the TOU price system is 15 minutes. For this reason, the STLF of an office building is performed for 15 minutes. Usually, the STLF is carried out from hours to weeks. Seconds or minutes to several hours are referred to as Very Short-Term Load Forecasting (VSTLF) [19], [20]. Therefore, we propose a VSTLF method for an office building.

In this paper, we propose the VSTLF method based on a pattern ratio. To develop the proposed method, electric demand by LG Electronics is analyzed. The proposed method is compared to the conventional method, which uses linear regression with temperature. To verify the VSTLF method, we used a forecasted electric demand of LG Electronics.

\section{Electric Demand Analysis of an Office Building}

We use 2012 to 2014 data from LG Electronics in Seoul. The LG electronics building is divided by research and experimental divisions. The electric demand of the research division is greatly influenced by an electric heat pump (EHP). In the experimental division, experimental equipment such as calorimeter strongly influences electric demand. The electric demand for Saturdays and holidays are not applied to measure a peak load under the TOU price system. These are excluded from the VSTLF. The time unit of the TOU price system is 15 minutes. Therefore, VSTLF is performed every 15 minutes.

When analyzing the electric demand pattern in terms of the days of the week, we find that there is no pattern as shown in Fig. 1. The electric demand from Monday to Friday has a similar pattern. The electric demand for Monday from 0:00 to 5:00 is a bit lower than for other days. However, this does not matter because there is no chance of obtaining a peak load at dawn. Therefore, clustering by days of the week should not greatly help to increase the accuracy. When considering seasonal change, our analysis of electric demand pattern shows that electric demand is related to season from Fig. 2. There is a correlation between electric demand and season. Because of air conditioners, peak load is highest in the summer season. The electric demand pattern for the summer has two noticeable small valleys in comparison with the other seasons. From the winter season graph in Fig. 2, the peak load is smoother than the other seasons. The electric demand of spring and fall has a similar shape. As a result, electric demand is relative to season. Finally, the relationship between electric load and temperature has been examined. Fig. 3 shows that there is a correlation between temperature and electric demand with a different relationship every 15 minutes. Electric demand during office hours, which is from 9:00 am to 6:00 pm, has a stronger correlation with temperature than during dawn hours. We concluded that electric demand is related to season and temperature. Therefore, we use this result to predict electric demand.
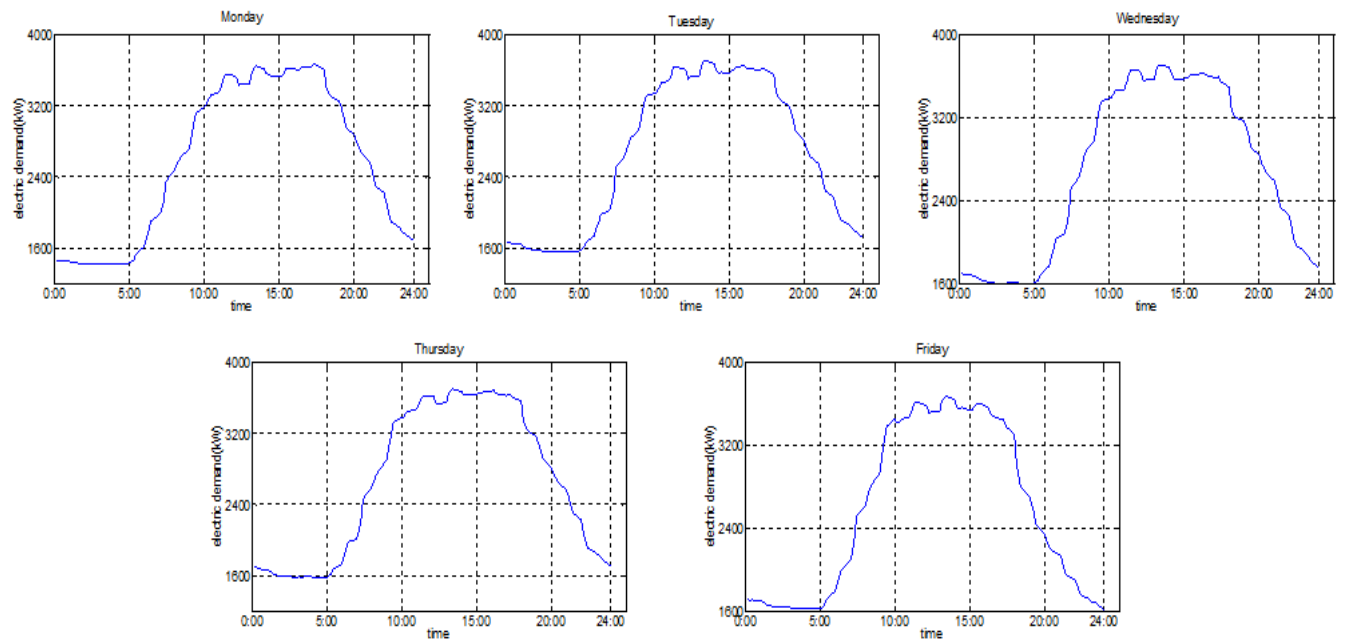

Fig. 1. Electric demand pattern for the days of the week. 

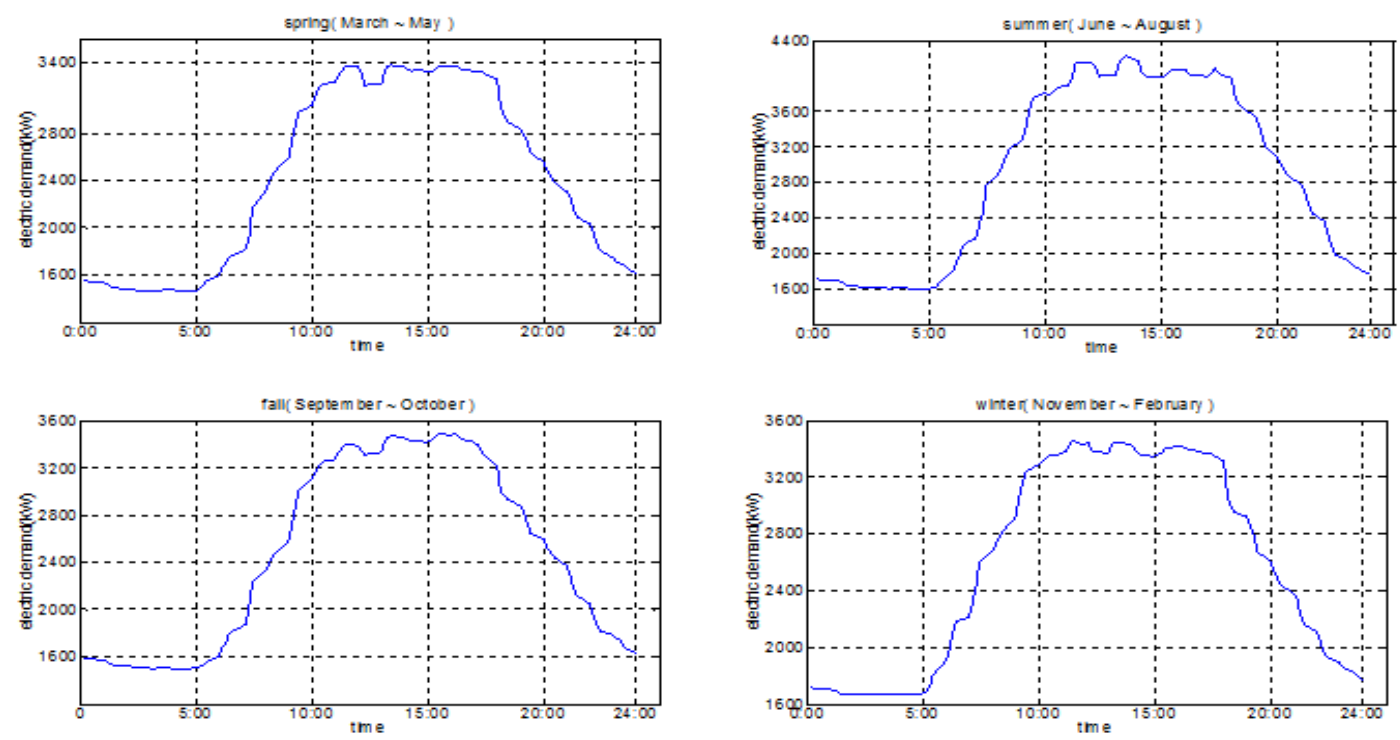

Fig. 2. Electric demand pattern of each season.
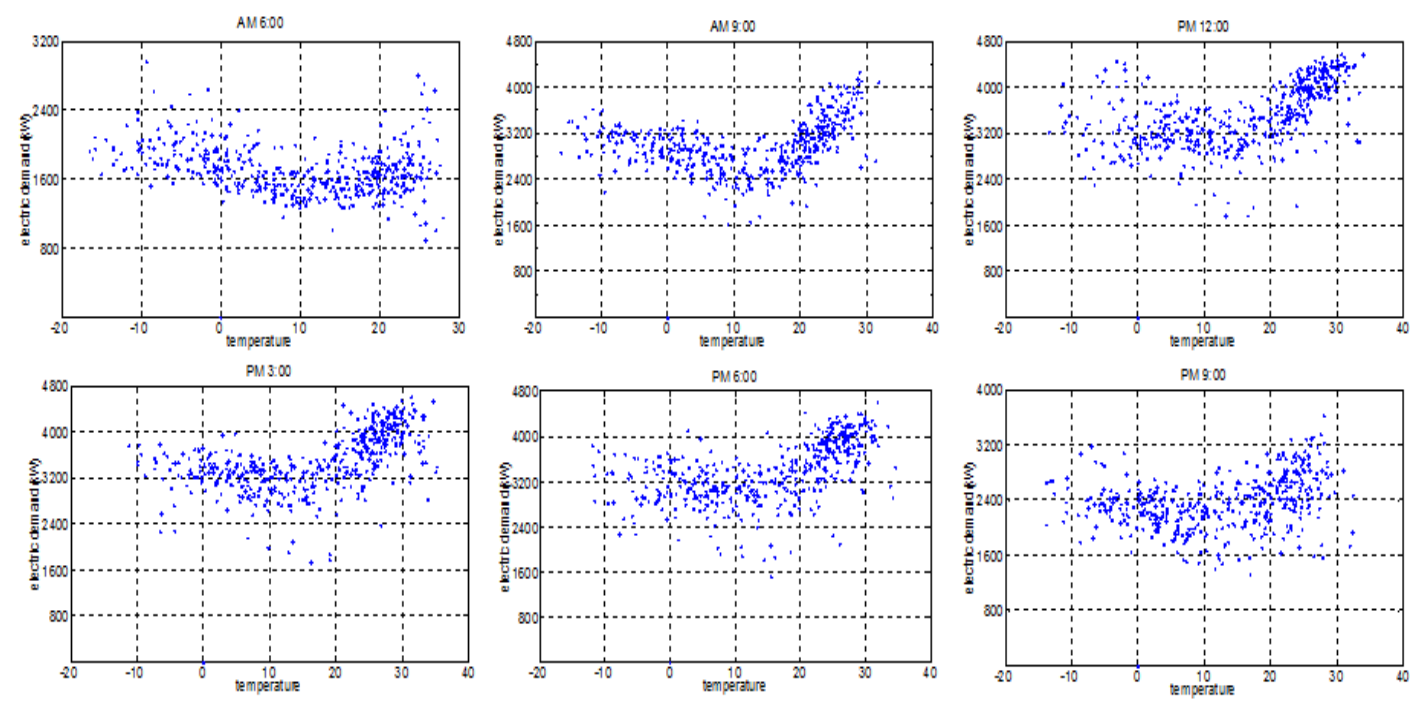

Fig. 3. Correlation between electric demand and temperature.

The electric demand of an office building is greatly influenced by unexpected variables in comparison with a large power system. Because the electric demand pattern of an office building is not smooth, it is very difficult to fit the function. Therefore, we carried out VSTLF based on a pattern ratio.

\section{VSTLF Method}

We describe the VSTLF method with two algorithms. A method that uses a correlation between electric demand and temperature is exhibited in 3.1. In 3.2, the proposed method based on a pattern ratio is discussed.

\subsection{Linear regression with temperature}

Linear regression has broadened the use of the relationship between electric demand and temperature 
[6], [10]-[15], [19]. This method is conventional. Specially, the STLF of a building considers the heating and air conditioning systems because the electric demand for these systems is heavy on the total electric demand. In Fig. 3, we set a reference point and then we fit two linear equations like Fig. 4. We decide on a reference point by using a genetic algorithm (GA). Based on the reference point, we calculate the approximate two linear equations by the least squares method every 15 minutes. In this process, we remove an outlier point and then derive a reference point. As a result, 96 reference points and 192 linear equations are created. Next, we eliminate temperature-sensitive from the electric demand. We calculate the average electric demand pattern by the following equation:

$$
L_{p}=L_{m}+\alpha *\left(T_{m}-T_{r}\right)
$$

$\mathrm{L}_{\mathrm{p}}$ is the average electric demand removed an effect of temperature for 30 days. The 30 days is composed of only weekdays. $\mathrm{Lm}$ is the average electric demand, which is measured for 30 days. $\alpha$ has a different value every 15 minutes and is a gradient from the linear equation that is calculated earlier. $\mathrm{T}_{\mathrm{m}}$ are measured temperatures for 30 days and $\mathrm{T}_{\mathrm{r}}$ are temperatures of the reference point:

$$
L_{f}=L_{p}-\alpha *\left(T_{f}-T_{r}\right)
$$

In this method, we need $\mathrm{T}_{\mathrm{f}}$, which is a prediction of temperature every 15 minutes. Because forecasting is performed one more time, there is growing uncertainty. This method needs large amounts of data. Otherwise, an error in forecasting increases. Therefore, mass storage devices are essential.
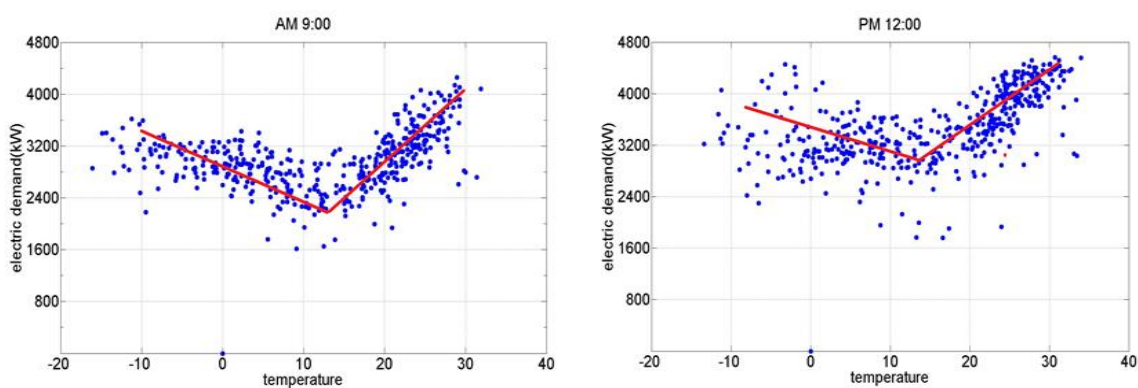

Fig. 4. Fitting a linear equation using a genetic algorithm.

\subsection{The proposed method based on a pattern}

In this section, we proposed a VSTLF method based on a pattern ratio. As mentioned earlier, it is difficult to predict electric demand of an office building using a function due to an unexpected event. The main idea of this method is that the change of electric consumption for only a brief time is very small. This idea was developed by the following calculation. The average electric consumption change for 15 minutes is $19.59 \mathrm{kWh}$ and the average electric consumption is $858.09 \mathrm{kWh}$. This was converted into a percentage of $2.28 \%$. Therefore, we assume that the measured electric consumption just before forecasting equals the electric consumption for the next 15 minutes. The proposed method is based on a pattern ratio and is performed with the following equation:

$$
\begin{aligned}
& p_{r}=\frac{L_{m}(t)}{L_{P}(t+15)}=\frac{L_{f}(t+15)}{L_{p}(t+15)} \\
& L_{f}(t+k)=p_{r} * L_{p}(t+k)
\end{aligned}
$$

$\mathrm{P}_{\mathrm{r}}$ is named the pattern ratio. $\mathrm{t}$ is at a forecasting time. $\mathrm{Lm}$ is as mentioned in $3.1 . \mathrm{L}_{\mathrm{p}}$ is the average 
electric demand for a certain period such as 10, 20, or 30 days just before the VSTLF.

Table 1. Characteristics of the VSTLF methods

\begin{tabular}{lll}
\hline & Input & Operation time \\
\hline $\begin{array}{l}\text { Conventional method } \\
\text { (linear regression with temperature) }\end{array}$ & Electric demand and temperature for at least 1 year & Several minutes \\
$\begin{array}{l}\text { The proposed method } \\
\text { (based on a pattern ratio) }\end{array}$ & Electric demand for a few days & Few seconds \\
\hline
\end{tabular}

The advantage of this method is that mass storage devices are not needed because the VSTLF is available by the electric consumption data for a few days. Although there is single day data, this method can predict electric demand. Also, the input of this method is only the measured electric consumption for a few days. There is no need for temperature data. In addition, the operation time is very short. Thus, this method is very suitable to VSTLF. However, the error of VSTLF increases further away from a forecasting time. Therefore, this method is effective for a very short term. The characteristic of these methods are summarized in Table 1.

\section{Case Study}

The results for the VSTLF case study from 2012 to 2014 are summarized in Table 2. We compare the linear regression method with temperature and the pattern ratio method using LG data. Fig. 5 shows that the electric demand pattern of the proposed method is a more similar measured value than the conventional method. Mean Absolute Percentage Error (MAPE) is an accuracy index of VSTLF. Because the electric demand of a small power system such as an office building is greatly influenced by unpredictable factors, MAPE is greater than the large power system [3]-[10]. In Table 2, we demonstrate that the method based on a pattern ratio has a better performance. In addition, the method based on a pattern ratio is simpler than a linear regression. Therefore, the proposed method based on a pattern ratio is suited for VSTLF.

\section{Conclusions}

In this paper, a short-term load forecasting every 15 minutes was proposed to support efficient and economical use of electricity in an office building. A basic concept of the proposed method is that electric consumption for 15 minutes does not change rapidly. Therefore, we predict electric demand using a pattern ratio. The proposed method has been shown to better perform than the conventional method using linear regression through simulation. MAPE of the proposed method is $9.0973 \%$, while MAPE of the conventional method is $9.4533 \%$. The biggest benefit of the proposed method is that the input is only the electric consumption for a few days. Even though this method appears very simple, the performance is better than the conventional method. Also, this method can work well in the abnormal condition by reflecting electric demand of last 15 minutes. In the case of a large-scale office building, BEMS is available to collect and store data such as temperature and humidity. In this case, we could predict electric demand by a more delicate method. If there is no BEMS in an office building, the proposed method is very useful. Based on the VSTLF method, BEMS of an office building can be the subject for future research.
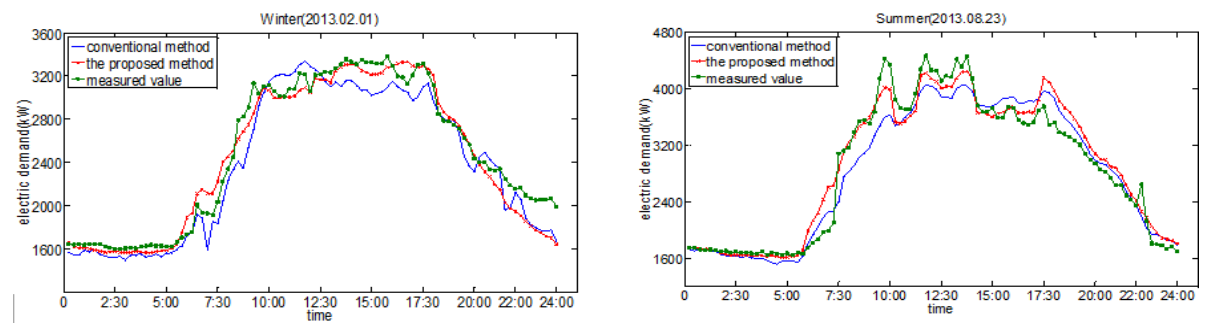

Fig. 5. Results of the VSTLF methods. 


\section{Acknowledgements}

This work was supported by the Global Excellent Technology Innovation (20132010101890) of the Korea Institute of Energy Technology Evaluation and Planning (KETEP), granted financial resource from the Ministry of Trade, Industry \& Energy, Republic of Korea.

\section{References}

[1] Leadbetter J, Swan L. Battery storage system for residential electricity peak demand shaving. Energy and Buildings, 2012; 55:685-692.

[2] Kim CH. Korea Short-Term Load Forecasting by TBATS model. Research report. Korea Energy Economics Institute, 2013.

[3] Zhang G, Patuwo BE, Hu MY. Forecasting with artificial neural networks: The state of the art. International Journal of Forecasting, 1998; 14:35-62.

[4] Darbellay GA, Slama M. Forecasting the short-tern demand for electricity. International Journal of Forecasting, 2000; 16:7183.

[5] Taylor W, de Menezes LM, McSharry PE. A comparison of univariate methods for forecasting electricity demand up to a day ahead. International Journal of Forecasting, 2006; 22:1-16.

[6] Papalexopoulos AD, Hesterberg TC. A regression-based approach to short-term system load forecasting. IEEE Tranaction Power Systems, 1990; 5(4):1535-1547.

[7] Charytoniuk W, Chen MS, Van Olinda P. Nonparametric regression based short-term load forecasting. IEEE Transaction Power Systems, 1998; 13(3):725-730.

[8] Amjady N. Short-term hourly load forecasting using time-series modeling with peak load estimation capability. IEEE Transaction Power Systems, 2001; 16(3):498-505.

[9] Huang SJ, Shih KR. Short-term load forecasting via ARMA model identification including non-gaussian process considerations. IEEE Transaction Power Systems, 2003; 18(2):673-679.

[10] Paparoditis E, Sapatinas T. Short-term load forecasting: the similar shape functional time-series predictor. IEEE Transaction Power Systems, 2013; 28(4):3818-3825.

[11] Penya YK, Borges CE, Fernandez I. Short-term load forecasting in non-residential buildings. In: Proc. 10th IEEE Region 8 Conference (AFRICON), 2011:1-6.

[12] Fernandez I, Borges CE, Penya YK. Efficient building load forecasting. In: Proc. 16th IEEE International Conference on Emerging Technologies and Factory Automation (ETGA), 2011:1-8.

[13] Borges CE, Penya YK, Fernandez I. Optimal combined short-term building load forecasting. In: Proc. IEEE PES Innovative Smart Grid Technologies Asia (ISGT), 2011:1-7.

[14] Brown M, Barrington-Leigh C, Brown Z. Kernel regression for real-time building energy analysis. Journal of Building Performance Simulation, 2012; 5(4):263-276.

[15] Lim JH, Kwon OS, Song KB, Park JD. Short-term load forecasting for educational buildings with temperature correlation. In: Proc. 4th IEEE International Conference on Power Engineering, Energy and Electrical Drives, 2013:405-408.

[16] Haben S, Ward J, Greetham DV, Singleton C, Grindrod P. A new error measure for forecasts of household-level, high resolution electrical energy consumption. International Journal of Forecasting, 2014; 30:246-256.

[17] Chaouch M. Clustering-based improvement of nonparametric functional time series forecasting: Application to intra-day household-level load curves. IEEE Transaction Smart Grid, 2014; 5(1):411-419.

[18] Weijie M, Chung CY, Ting W, Huazhang H. Electric load forecasting for large office building based on radial basis function neural network. In: Proc. PES General Meeting, 2014:1-5.

[19] Hernandez L, Baladron C, Aguiar JM, Carro B, Sanchez-Esguevillas AJ, Lloret J, Massana J. A survey on electric power demand forecasting: future trends in smart grids, microgrids and smart buildings. IEEE Commun. Surv. Tutor, 2014; 16(3):1460-1495.

[20] Charytoniuk W, Mo-Shing C. Very short-term load forecasting using artificial neural networks. IEEE Trans. Power Syst, 2000; 15(1):725-730. 